\title{
Namib Desert Region Vegetation Database
}

\author{
Norbert Jürgens \& Gerhard Muche
}

\begin{abstract}
Since 1980 many observations of the vegetation in the dry regions of Namibia and South Africa have been recorded in the database of Namib Desert Region Vegetation Database (GIVD ID AF-00-007). All relevés were recorded using a standard size of $1000 \mathrm{~m}^{2}$ and include spatial reference data, habitat information and all observed species occurrences with cover and (partly) abundance data. Worth mentioning is that the database has been sampled over a period of several years thereby including different seasons as well as dry and wet years. Many relevés do not show the entire local species pool at the time of documentation. The interpretation of therophytic, geophytic and hemicryptophytic species must take this into consideration. Conditions of strongly grazed plant individuals in particular during dry seasons may have resulted in some inconsistencies in plant identifications, e.g. Stipagrostis hirtigluma subsp. hirtigluma versus S. uniplumis var. intermedia.
\end{abstract}

Keywords: desert; diversity; global change.

\section{Namib Desert Region Vegetation Database}

Scope: Vegetation and environmental data in Namibia, western South Africa and Angola

Status: ongoing capture

Period: $1980-2010$

Database manager(s): Norbert Jürgens (norbert.juergens@t-online.de); Gerhard Muche (gerhard.muche@uni-hamburg.de)

Owner: Norbert Jürgens

Web address: http://www.biota-africa.org

Availability: according to a specific agreement

Database format(s): MS Access, BIOTABase

Online upload: no

Online search: no

Publication: [NA]

Plot type(s): normal plots; time series

Export format(s): Excel, CSV file, Cornell condenses format, Canoco environment data

Non-overlapping plots: 10,902

Total plot observations: 10,952

Plot-size range: $10,000-10,000 \mathrm{~m}^{2}$

Completeness: [NA]

Valid taxa: 1,827

Countries: AO: $2.4 \%$; BW: $0.1 \%$; NA: $50.0 \%$; ZA: $47.6 \%$

Forest: $0 \%$ - Non-forest: aquatic: 0\%; semi-aquatic: 0\%; arctic-alpine: 0\%; natural: $64 \%$; semi-natural: $36 \%$; anthropogenic: $0 \%$

Guilds: all vascular plants: $100 \%$

Environmental data: altitude: 35\%; slope aspect: 35\%; slope inclination: $33 \%$; soil depth: $30 \%$; surface cover other than plants (open soil, litter, bare rock etc.): $25 \%$; soil $\mathrm{pH}: 25 \%$

Performance measure(s): presence/absence only: $28 \%$; cover: $72 \%$

Geographic localisation: GPS coordinates (precision $25 \mathrm{~m}$ or less): $42 \%$; small grid (not coarser than $10 \mathrm{~km}$ ): $43 \%$; political units or only on a coarser scale (>10 km): $15 \%$

Sampling periods: 1980-1989: 14.0\%; 1990-1999: 50.6\%; 2000-2009: 24.3\%; 2010-2019: 0.1\%; unknown: 11.0\% Information as of 2012-07-12; further details and future updates available from http://www.givd.info/ID/AF-00-007

Norbert Jürgens (norbert.juergens@t-online.de), Gerhard Muche* (gerhard.muche@uni-hamburg.de)

Biodiversity, Evolution and Ecology of Plants (BEE), Biocentre Klein Flottbek and Botanical Garden, University of Hamburg,

Ohnhorststr. 18, 22609 Hamburg, GERMANY

*Corresponding author 